ARTICLE

\title{
The docking of synaptic vesicles on the presynaptic membrane induced by $\alpha$-synuclein is modulated by lipid composition
}

\author{
Wing K. Man', Bogachan Tahirbegi ${ }^{2}$, Michail D. Vrettas (10 ${ }^{3}$, Swapan Preet ${ }^{1}$, Liming Ying (i) ${ }^{4}$, \\ Michele Vendruscolo (1) ${ }^{1}$, Alfonso De Simone $\mathbb{D}^{3,5 \times} \&$ Giuliana Fusco ${ }^{1 凶}$
}

$\alpha$-Synuclein $(\alpha S)$ is a presynaptic disordered protein whose aberrant aggregation is associated with Parkinson's disease. The functional role of $\alpha S$ is still debated, although it has been involved in the regulation of neurotransmitter release via the interaction with synaptic vesicles (SVs). We report here a detailed characterisation of the conformational properties of $\alpha S$ bound to the inner and outer leaflets of the presynaptic plasma membrane (PM), using small unilamellar vesicles. Our results suggest that $\alpha S$ preferentially binds the inner PM leaflet. On the basis of these studies we characterise in vitro a mechanism by which $\alpha \mathrm{S}$ stabilises, in a concentration-dependent manner, the docking of SVs on the PM by establishing a dynamic link between the two membranes. The study then provides evidence that changes in the lipid composition of the PM, typically associated with neurodegenerative diseases, alter the modes of binding of $\alpha \mathrm{S}$, specifically in a segment of the sequence overlapping with the non-amyloid component region. Taken together, these results reveal how lipid composition modulates the interaction of $\alpha \mathrm{S}$ with the PM and underlie its functional and pathological behaviours in vitro.

\footnotetext{
${ }^{1}$ Centre for Misfolding Diseases, Department of Chemistry, University of Cambridge, Cambridge CB2 1EW, UK. ${ }^{2}$ Department of Chemistry, Imperial College London, Molecular Sciences Research Hub, White City Campus, London W12 OBZ, UK. ${ }^{3}$ Department of Pharmacy, University of Naples "Federico II", Naples 80131, Italy. ${ }^{4}$ National Heart and Lung Institute, Imperial College London, Molecular Sciences Research Hub, White City Campus, London W12 OBZ, UK.

${ }^{5}$ Department of Life Sciences, Imperial College London, South Kensington SW7 2AZ, UK. ${ }^{凶}$ email: alfonso.desimone@unina.it; gf203@cam.ac.uk
} 
-Synuclein $(\mathrm{aS})$ is a $14 \mathrm{kDa}$ protein whose aggregation is strongly linked with Parkinson's disease (PD) and other neurodegenerative disorders collectively known as synucleinopathies, which include dementia with Lewy bodies and multiple system atrophy ${ }^{1-4}$. Aggregates of aS are major components of intraneuronal inclusions known as Lewy bodies ${ }^{5}$. Genetic links also exist between $\alpha \mathrm{S}$ and familial forms of early onset PD, including mutations, duplications and triplications of the aS encoding gene ${ }^{3}$.

Although the function of $\alpha \mathrm{S}$ is still debated ${ }^{6}$, its prevalence at the presynaptic termini indicates that it may be involved in synaptic plasticity ${ }^{7}$ and learning 8 . More specifically, a large body of evidence exists about a role of $a S$ in the regulation of the homoeostasis of synaptic vesicles (SVs) during neurotransmitter release $^{9-14}$, including contexts requiring intense neuronal activity $^{15}$. aS shows binding affinity for SVs in vitro and strongly colocalises with SVs in synaptosomes in the presence of calcium ions $^{16}$. Upon the binding of SVs, aS has a tendency to promote their clustering ${ }^{12-14,17}$, a process that has been associated with the maintenance of SV pools at the synaptic termini ${ }^{11,18-20}$. Additionally, $\alpha \mathrm{S}$ has been shown to influence the regulation of the vesicle trafficking from the endoplasmic reticulum (ER) to the Golgi $^{13,20}$, and to localise at mitochondrial membranes, where it has been proposed to mitigate the effects of oxidative stress ${ }^{21-24}$. All these putative functions by aS require its binding to biological membranes ${ }^{25,26}$, a central interaction that defines the relevant biological form of $\alpha \mathrm{S}$ in vivo ${ }^{27}$ and influences the kinetics of its aggregation $11,28,29$, as well as the toxicity of its aggregates $1,30,31$.

The characterisation of the binding mechanism of aS with synaptic membranes is therefore crucial to clarify its biological properties under physiological and pathological conditions. The intrinsic structural disorder of $a \mathrm{~S}$ in the cytosol ${ }^{32}$, however, is partially retained in its membrane-bound state, making it challenging to study the mechanism of membrane binding as well as the conformational properties of its membrane-associated forms. When bound with lipid bilayers, aS becomes enriched in amphipathic $a$-helical structure, a conformational feature promoted by seven imperfect sequence repeats in the region spanning residues $1-90^{33-37}$. These modular sequences provide $\alpha \mathrm{S}$ the plasticity to bind a large variety of lipid membranes via multiple binding modes ${ }^{35}$ and adopting different structural topologies such as broken ${ }^{36,38}$ and fully extended $\alpha$-helices ${ }^{27,39}$.

We here studied the conformational properties of aS upon binding with the inner (cytosolic) and outer (extracellular) leaflets of the presynaptic membrane (PM). The results indicate a considerable preference to bind the inner PM leaflet (IPM), where aS induces in a concentration-dependent manner a stabilisation of the docking of SVs. The underlying 'double-anchor' mechanism is promoted by distinctive structural and topological properties of $a S$ at the surface of IPM. The data then show that changes in the lipid composition of the PM, of the type occurring in neurodegenerative conditions, alter the binding and conformational properties of $\alpha \mathrm{S}$, specifically in a segment of the sequence that overlaps substantially with the non-amyloid component (NAC) region.

Taken together these results reveal how the conformational properties of $\alpha \mathrm{S}$ at the inner and outer leaflets of PM are strongly influenced by the composition of the lipid membrane, and regulate its behaviour under physiological and pathological conditions.

\section{Results}

Structural properties of $\alpha \mathrm{S}$ at the inner and outer leaflets of the presynaptic membrane. We investigated the binding of $\mathrm{N}$ terminally acetylated $\alpha \mathrm{S}$, the most common form of the protein

\begin{tabular}{|lllll|}
\hline $\begin{array}{l}\text { Table } 1 \text { Molar fractions of the lipid bilayers used in } \\
\text { this work. } \\
\end{array}$ IPM & OPM & IPM-GMs & OPM-GMs \\
\hline PC & 0.14 & 0.253 & 0.112 & 0.2 \\
PE & 0.219 & 0.115 & 0.176 & 0.091 \\
PS & 0.101 & - & 0.080 & - \\
PI & 0.052 & - & 0.041 & - \\
PIPS & 0.015 & - & 0.012 & - \\
Cholesterol & 0.458 & 0.464 & 0.367 & 0.368 \\
Sphingomyelin & 0.016 & 0.062 & 0.012 & 0.049 \\
Cerebrosides & - & 0.077 & - & 0.061 \\
GM1 & - & 0.015 & 0.100 & 0.115 \\
GM3 & - & 0.015 & 0.100 & 0.115 \\
\hline
\end{tabular}

in vivo ${ }^{40}$, with lipid bilayers that mimic the composition of the inner (IPM) and the outer (OPM) leaflets of PM. These two leaflets share common lipid components, including phosphatidylcholine (PC), phosphatidylethanolamines (PE), sphingomyelin and cholesterol (Table 1), while featuring distinctive ones, such as phospho-L-serine (PS), phosphatidylinositol (PI) and phosphatidylinositol phosphates (PIPs), which are specific to IPM, and gangliosides (GMs) and cerebrosides, which are specific to $\mathrm{OPM}^{41,42}$

IPM and OPM lipid mixtures were prepared in the form of small unilamellar vesicles (SUVs) with size distributions centred around $50 \mathrm{~nm}$, as measured using dynamic light scattering (DLS) (see Methods). The utilisation of this form of lipid assemblies enables a direct comparison of the present results with interaction studies previously performed with synaptic-like SUVs (SL-SUVs) composed of dioleoyl-phosphoethanolamine (DOPE), dioleoylphosphatidylcholine (DOPC) and dioleoyl-phosphatidylserine (DOPS) $)^{33}$ and cholesterol ${ }^{43}$.

We first examined the conformational basis of the interaction of aS with IPM and OPM using chemical exchange saturation transfer (CEST) experiments in solution nuclear magnetic resonance (NMR) spectroscopy (Fig. $1 \mathrm{a}-\mathrm{c})^{33,44-47}$. CEST has been shown to be an accurate probe of the equilibrium between membrane-unbound and membrane-bound states of $\alpha S^{17,33}$ that are, respectively, detectable and undetectable in solution NMR experiments. CEST is based on the selective saturation of NMRundetectable membrane-bound states using a continuous weak radiofrequency field at offsets that range up to $\pm 28 \mathrm{kHz}^{16,17,33}$. The saturation is then observed as an attenuation of the signal intensities in the NMR-detectable unbound state of the protein as a result of the exchange between bound and unbound conformations. In probing the interaction between aS and lipid vesicles at a residue-specific resolution, the current implementation of CEST offers advantages over methods based on the signal attenuation in ${ }^{1} \mathrm{H}-{ }^{15} \mathrm{~N}-\mathrm{HSQC}$ spectra. By saturating the protein resonances directly in the membrane-bound state, CEST probes exclusively the binding strength between individual residues of aS and the membranes, resulting in high sensitivity also at low lipid/ protein ratios, conditions under which protein or lipid aggregation can be minimised. In addition, CEST data are largely independent from side factors that may affect transverse relaxation of the protein during the experiment, such as for example conformational exchange in the millisecond timescale.

${ }^{1} \mathrm{H}-{ }^{15} \mathrm{~N}$ CEST measurements employing two different saturation frequencies $(350 \mathrm{~Hz}$ in Figs. $1 \mathrm{a}, \mathrm{b}$ and $\mathrm{S} 1$ and $170 \mathrm{~Hz}$ in Fig. S2) were carried out on a sample composed of $\alpha \mathrm{S}(300 \mu \mathrm{M})$ and IPM or OPM SUVs $(0.6 \mathrm{mg} / \mathrm{ml})$. The measured CEST profiles revealed a significant difference in the binding of aS with the two leaflets of the PM. In particular, the protein showed moderate binding affinity for IPM, with high saturation primarily found in 

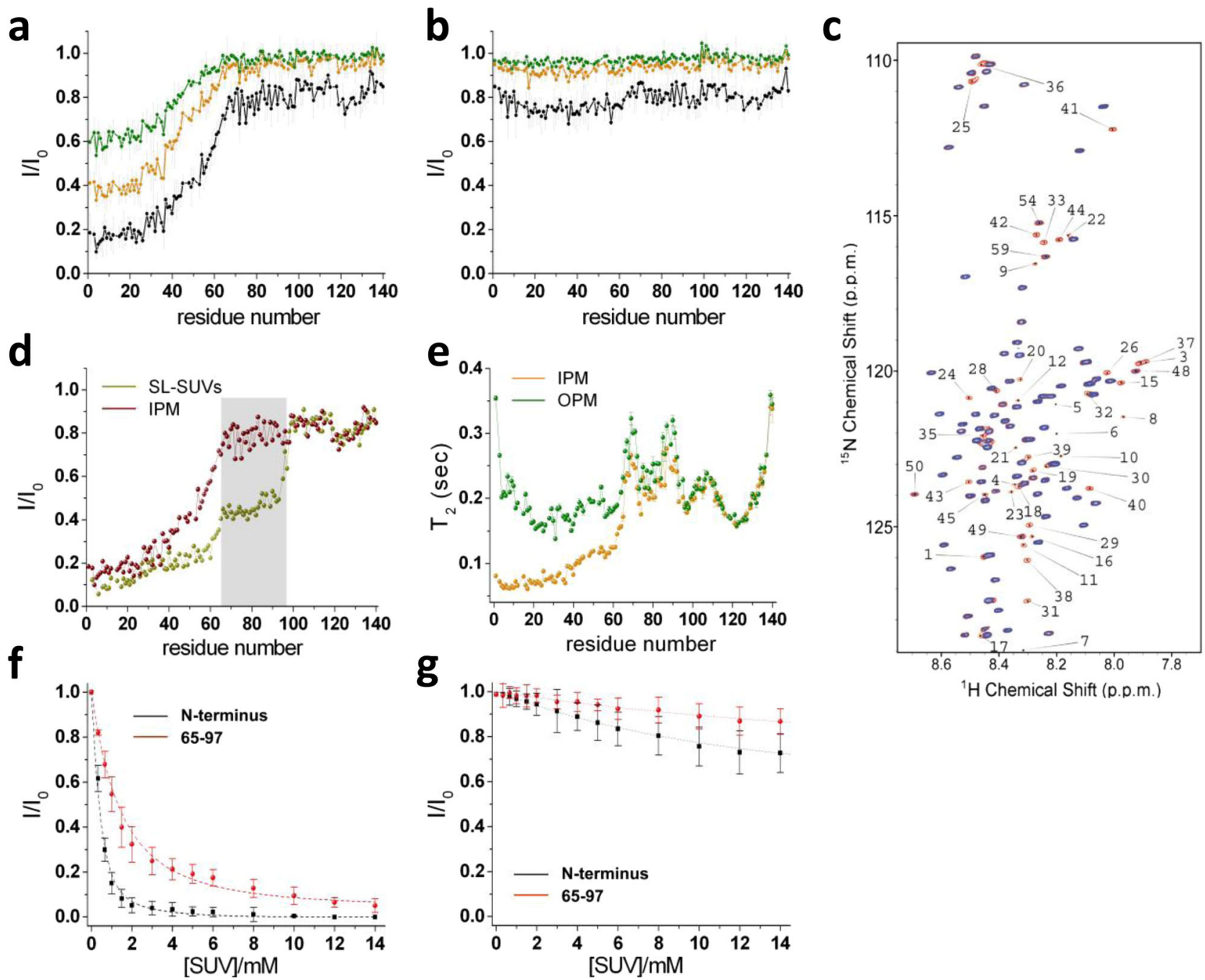

Fig. $1 \alpha$ S binds IPM more strongly than OPM. Interaction between $\alpha$ S and IPM (a) and OPM (b) monitored using NMR CEST. Spectra were aquired at $283 \mathrm{~K}$ in $20 \mathrm{mM}$ of phosphate buffer at $\mathrm{pH} 6.0$, at a ${ }^{1} \mathrm{H}$ frequency of $700 \mathrm{MHz}$, using a protein and SUV IPM concentrations of $300 \mu \mathrm{M}$ and $0.06 \%$ $(0.6 \mathrm{mg} / \mathrm{ml}$ ), respectively. NMR CEST profiles measured using a saturation bandwidth of $350 \mathrm{~Hz}$ (Fig. S2 for measurements performed with a bandwidth of $170 \mathrm{~Hz}$ ) and probing the interaction between $\alpha \mathrm{S}$ and IPM (a) or OPM (b). Black, orange and green lines refer to the averaged CEST profiles measured using offsets at $\pm 1.5, \pm 3.0$, and $\pm 5.0 \mathrm{kHz}$, respectively. Error bars report the standard deviation estimated on the triplicate measurements. c Representative ${ }^{1} \mathrm{H}-15 \mathrm{~N}-\mathrm{HSQC}$ CEST spectra of $\alpha \mathrm{S}$ in the presence of IPM measured using a $350 \mathrm{~Hz}$ continuous wavelength at offsets of $100 \mathrm{kHz}$ (red) and $1.5 \mathrm{kHz}$ (blue). d Comparison of the interaction of $\alpha \mathrm{S}$ with SL-SUVs 43 (yellow) and IPM (red) probed with NMR CEST profiles measured using a saturation bandwidth of $350 \mathrm{~Hz}$ and offsets of $\pm 1.5 \mathrm{kHz}$. Data for the SL-SUVs binding ${ }^{43}$ were measured at protein and lipid concentrations of $300 \mu \mathrm{M}$ and $0.06 \%(0.6 \mathrm{mg} / \mathrm{ml})$, respectively. Grey background highlights the significant difference in the saturation of the region spanning residues 65-97, resulting in populations of detached conformations for this region of $51 \%$ and $95 \%$ for SL-SUVS and IPM, respectively. e $T_{2}$ values from transverse relaxation measurements (experimental conditions as in $\mathbf{a}, \mathbf{b}$ ). Green and orange report $T_{2}$ values of $\alpha \mathrm{S}$ in the presence of OPM and IPM, respectively. Error bars report the $T_{2}$ fitting error. $\mathbf{f}-\mathbf{g}$ Binding curves of $\alpha \mathrm{S}$ to SUVs monitored via the signal attenuation of the peaks in the ${ }^{1} \mathrm{H}-15 \mathrm{~N}-\mathrm{HSQC}$ spectra of $\alpha \mathrm{S}$ (50 $\mu \mathrm{M}$ ) as a function of the concentration of SUVs. The signal attenuations have been averaged across the residues of the N-terminal region (black) and the region spanning residues 65-97 (red) and error bars report the standard deviation of these values. In the case of IPM, the fitting provided $K_{D}$ values of 5.2 $\mu M(L=14.3)$ and $88.9 \mu \mathrm{M}(L=10.3)$ for the $\mathrm{N}$-terminal (residues 1-25) and central (residues 65-97) region, respectively, whereas for OPM $K_{D}$ values resulted respectively $5933 \mu \mathrm{M}(L=5.7)$ and $13,689 \mu \mathrm{M}(L=6.1)$.

the acetylated $\mathrm{N}$ terminus of $\mathrm{\alpha S}$ (Fig. 1a). The binding strength for IPM SUVs of this region resulted to be similar to that observed in the case of SL-SUVs (Fig. 1d), where the $\mathrm{N}$ terminus has a role of anchor for the membrane binding by $\alpha S^{33,48}$. The saturation effects were found to decrease gradually in the residues following the N-terminal anchor, with a sharp transition observed in the region spanning residues 40-64 (Fig. 1a) leading to an almost complete loss of membrane affinity in the region 65-140. This finding therefore reveals that $\alpha \mathrm{S}$ adopts unusual conformational properties when bound to the surface of IPM. In particular the region 65-97, which overlaps in large part with the amyloidogenic
NAC region ${ }^{5,49}$, resulted to be mostly unbound to IPM surfaces up to the same level of the negatively charged region 98-140, which generally shows poor affinity for lipid membranes ${ }^{33,35}$ and detergent micelles ${ }^{38}$.

A different scenario was observed in the study of the aS interaction with OPM. In particular, CEST measurements resulted in very similar saturation profiles to those of the isolated $\alpha \mathrm{S}$ in solution ${ }^{33}$ (Figs. 1b, S1 and S2). When plotting the CEST profiles along the sequence, only the experiments performed using offsets at $\pm 1.5 \mathrm{kHz}$ and a saturation frequency of $350 \mathrm{~Hz}$ showed some very mild saturation levels in the region 
of residues 1-65 of the protein, but this effect resulted too weak to be detected at other offsets or using a saturation frequency of $170 \mathrm{~Hz}$.

Further details on the binding of $\alpha \mathrm{S}$ with IPM and OPM were obtained using experiments of ${ }^{15} \mathrm{~N}$ transverse relaxation (Fig. 1e). In the case of IPM, these measurements reported low $T_{2}$ values for the $\mathrm{N}$-terminal region, indicating strong local membrane interactions, and high values of $T_{2}$ in the regions 65-97 and 98-140, indicating negligible membrane binding. Conversely, in the case of OPM, ${ }^{15} \mathrm{~N}$ transverse relaxation experiments showed high values of $T_{2}$ throughout the membrane-binding region.

In order to quantify the binding affinity for IPM and OPM, we analyzed the signal attenuation in ${ }^{1} \mathrm{H}_{-}{ }^{15} \mathrm{~N}-\mathrm{HSQC}$ spectra of $\alpha \mathrm{S}$ $(50 \mu \mathrm{M})$ in the presence of increasing amounts of SUVs (from 0 to $14 \mathrm{mM}$ ), and averaged these data across two independent protein regions having distinct roles in the interaction with biological membranes, namely the $\mathrm{N}$-terminal anchor (residues 1-25) and the region spanning residues 65-97. The resulting binding curves were fitted using a quadratic expression ${ }^{50}$ (Eq. 1) that provides both the dissociation constant, $K_{D}$, and the number of lipid molecules, $L$, interacting with a single aS molecule:

$$
\chi_{B}=\frac{\left([\alpha S]+\frac{[S U V s]}{L}+K_{D}\right)-\sqrt{\left([\alpha S]+\frac{[S U V s]}{L}+K_{D}\right)^{2}-\frac{4[S U V s][\alpha S]}{L}}}{2[\alpha S]}
$$

where $\chi_{B}$ is the fraction of bound protein estimated as $\left(1-\frac{I}{I_{0}}\right)$ for each residue and averaged across the analyzed protein region. The results indicated that the $\mathrm{N}$ terminus of $\alpha \mathrm{S}$ interacts strongly with IPM $\left(K_{D}=5.2 \mu \mathrm{M}\right.$ and $\left.L=14.3\right)$ whereas the region $65-97$ has a nearly 18 -fold decrease in affinity for these membranes $\left(K_{D}=\right.$ $88.9 \mu \mathrm{M}$ and $L=10.3$ ) (Fig. $1 \mathrm{f}$ ). By contrast, aS binding with OPM (Fig. $1 \mathrm{~g}$ ) was found to be very weak for both the $\mathrm{N}$ terminus $\left(K_{D}=5933 \mu \mathrm{M}\right.$ and $\left.L=5.7\right)$ and for the region $65-97\left(K_{D}=\right.$ $13,689 \mu \mathrm{M}$ and $L=6 \cdot 1)$. A significant difference in the interaction with IPM and OPM was also detected using circular dichroism (CD), which probes the conformational changes from disorderedunbound to helical membrane-bound states of aS (Fig. S3).

Mechanism by which $\alpha \mathrm{S}$ assists SV docking to IPM. The study of the binding modes of aS with IPM indicated a very weak membrane affinity in the region spanning residues 65-97 (Fig. 1f) when compared with the interaction with SL-SUVs (Fig. 1d). In particular, conformations featuring a membrane-unbound region 65-97 were found to be $95 \%$ in the case of IPM and $51 \%$ in the case of SL-SUVs ${ }^{43}$. This significant difference is expected to result in different behaviours adopted by $a \mathrm{~S}$ at the surface of these two membrane types, as indeed the membrane affinity of the region 65-97 is a key determinant of its biological properties ${ }^{17,33}$. This region is indeed a crucial driver of the affinity of aS for acidic membranes ${ }^{33}$ and plays a role in the promotion of SV clustering via a 'double-anchor' mechanism ${ }^{17}$ in which the N-terminal region (first anchor) and the region 65-97 (second anchor) bind simultaneously two different vesicles up to a distance of $150 \AA^{17}$.

The analysis of the conformational properties at the surface of IPM indicates that, upon binding via the N-terminal anchor, the region spanning residues 65-97 remains largely available to engage in interactions with other molecules and organelles, including SVs. This finding suggests that the conformations adopted by the IPM-bound aS are particularly active to stabilise the docking of SV on the IPM surface (Fig. 2a). The stronger affinity of the region 65-97 to bind the membrane component of SVs as respect to IPM (Fig. 1d) also indicates that this protein segment would preferentially interact with SVs over IPM, thereby suggesting a topological preference in the double-anchor mechanism.

To generate quantitative analysis of the stabilisation of SV docking onto the PM by $\alpha \mathrm{S}$, we used total internal reflection fluorescence (TIRF) microscopy, which enabled to sample the nature of hundreds of docking events in individual experiments (Fig. 2b, c). TIRF imaging was carried out by fluorescently labelling SL-SUVs using $0.1 \%$ Topfluor ${ }^{\circledast}$ PC lipids and by forming an IPM lipid bilayer on glass surfaces via overnight incubation at $4{ }^{\circ} \mathrm{C}$ of IPM SUVs (Methods). As the focal plane of our TIRF setup extends up to $150 \mathrm{~nm}$ from the glass surface (Fig. 2c), the resulting images capture SL-SUVs that are in focus when they dock or are in close proximity of the IPM lipid bilayer. Vesicles in the bulk solution remain out of focus and their blurred bright dot images can be filtered out during the image processing (see Methods).

TIRF experiments were performed by incubating fluorescently labelled SL-SUVs in glass wells coated with IPM lipid bilayers. TIRF image sequences were recorded for $300 \mathrm{~s}$ with a frame rate of 25 frames per second (Methods) after a photobleaching step (160 s) that removed the contribution of vesicles docked prior to the sampling. Measurements were first performed in the absence of $\alpha \mathrm{S}$ and subsequently by adding increasing concentrations of the protein in the same well. The results show that by maintaining a constant concentration of SL-SUVs in the well $(2 \mu \mathrm{M})$ the number of vesicles docking onto the IPM surface increases in the presence of increasing concentrations of aS (Fig. 2d). In particular, under these experimental conditions, the strongest increase in SL-SUVs docking was observed in measurements made using $10 \mu \mathrm{M} \alpha \mathrm{S}$, with $26.8 \pm 2.1$ vesicles sampled per frame compared to $11.5 \pm 2.3$ observed in the absence of $\alpha \mathrm{S}$. An additional increase to $37.6 \pm 6.4$ vesicles in the images was measured when aS concentration was $100 \mu \mathrm{M}$. In addition to modifying the propensity of docking, our experiments indicated that aS alters the time spent by SL-SUVs onto IPM surfaces (Fig. 2e). In particular, by calculating the autocorrelation function (ACF) of the vesicles in the TIRF focal plane (Fig. S4), we estimated the residence time of docked SL-SUVs increasing from $440 \pm 8$ to $900 \pm 9 \mathrm{~ms}$ in the presence of 0 and $10 \mu \mathrm{M} \alpha \mathrm{S}$, respectively. An additional 10\% increase of the residence time was found when the concentration of $\alpha \mathrm{S}$ was set to $100 \mu \mathrm{M}$. The observed concentration dependence suggests that multiple aS molecules can simultaneously contribute to the stabilisation of the docking of a single vesicle.

Changes in the PM composition modulate the properties of $\alpha \mathrm{S}$ at the surface of IPM and OPM. In some neurodegenerative conditions, increased amounts of GMs in neuronal membranes have been observed ${ }^{51}$. Alterations in the GM fractions were found in analyses of Alzheimer's disease brains, resulting in an increase of GM clustering at presynaptic neuritic terminals ${ }^{52}$. Analyses of brains from Alzheimer's patients also found high concentration of GMs in detergent-resistant membrane fractions from the frontal cortex and the temporal cortex ${ }^{53}$ as well as in lipid rafts ${ }^{54}$. In addition, age-dependent mechanisms were also shown to induce increase in the density of GMs in some neuronal populations ${ }^{52}$. An increase in the GM content of the cellular membrane is also associated with enhanced toxicity of misfolded protein oligomers ${ }^{55}$. aS has significant propensity for in vitro binding of SUVs containing monosialotetrahexosylganglioside $(\mathrm{GM} 1)^{56,57}$ as well as liquid-ordered lipid domains of cytoplasmic membranes that are rich in $\mathrm{GM}^{58}$. It has also been proposed that elevated concentrations of GM1 in lipid rafts favour the cell internalisation of $a S^{59}$. The effect of GMs on the aggregation properties of aS are currently debated, with both inhibition ${ }^{56}$ or 

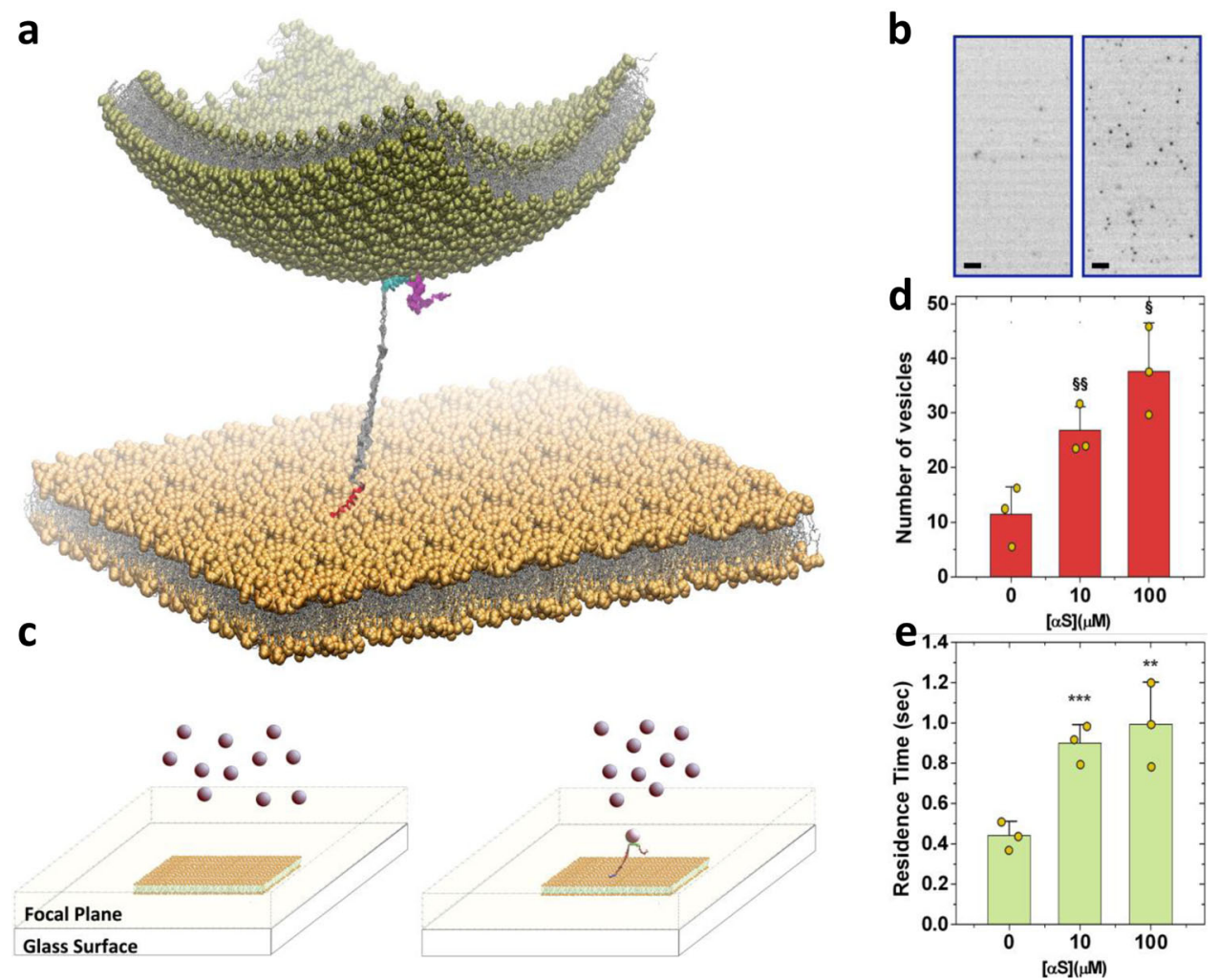

Fig. $2 \alpha \mathbf{S}$ mediates the docking of SVs to IPM. a Illustration of the mechanism by which $\alpha$ S tethers the outer leaflet of a SV (green vesicle) to the inner leaflet of a synaptic membrane (flat yellow membrane). A double-anchor conformation of $\alpha \mathrm{S}$ was modelled with the $\mathrm{N}$-terminal region (red) bound to the IPM and the region of residues 65-97 (cyan) tethering the SV. Both anchors are modelled as amphipathic $\alpha$-helices whereas the C-terminal region (residues 98-140, magenta) and the linker between the anchors (residues 26-64, grey) are in disordered conformations. $\mathbf{b}$ Example of TIRF images measured using $2 \mu \mathrm{M}$ of fluorescently labelled SL-SUVs and IPM deposited onto the glass surface. Images obtained using 0 and $10 \mu \mathrm{M}$ of $\alpha \mathrm{S}$ are shown on the left and right, respectively (scale bar, $2 \mu \mathrm{m}$ ). These representative images are part of 7500 frames acquired at $40 \mathrm{~ms}$ of exposure time in TIRF videos. Three TIRF videos per sample condition were recorded and analyzed to quantify the number of docked vesicles and their residence time (d, e respectively). c Scheme of the TIRF imaging employed in this study. The focal plane extends up to $150 \mathrm{~nm}$ from the glass surface where the IPM are deposited (yellow membrane). SL-SUVs (pink spheres) float in the bulk solution and come into focus when docked onto the IPM surfaces. The schematic view shows measurements made in the absence (left) and presence (right) of $\alpha \mathrm{S}$ and provide an example of how the double-anchor mechanism stabilises docked vesicles that are therefore imaged in the focal plane. $\mathbf{d}$ Statistical analysis of the number of docked vesicles on the IPM surface residing in the focal plane at different concentrations of $\alpha S$ and constant concentration of SUVs $(2 \mu \mathrm{M})$. The symbols $\S$ and $\S \S$, indicate $p$ values of 0.018 and 0.021 , respectively, calculated with the unpaired $t$ test using Welch's correction. Error bars report the standard deviation estimated on the triplicate measurements.

e Residence times of docked vesicles on the IPM surface at different concentrations of $\alpha \mathrm{S}$ and constant concentration of SUVs ( $2 \mu \mathrm{M})$. The symbols ** and ${ }^{\star \star \star}$, indicate $p$ values of 0.033 and 0.003 , respectively, calculated with the unpaired $t$ test using Welch's correction. Error bars report the standard deviation estimated on the triplicate measurements.

enhancement ${ }^{60,61}$ of the aggregation kinetics being observed depending on the experimental conditions.

Given these observations, we studied the modulation of the binding of the monomeric aS with IPM and OPM by an enrichment of the GM component (denoted as IPM-GMs and OPM-GMs, respectively). Under physiological conditions, GMs have an asymmetric distribution and partition primarily on the OPM side 62 . It is unclear, however, whether the perturbation of lipid homoeostasis observed in some neurodegenerative conditions results in an increase of GMs for both the inner and outer leaflets of the $\mathrm{PM}^{52}$. Our analysis showed that $\alpha \mathrm{S}$ has stronger affinity to bind OPM-GMs than OPM (Fig. 3a). Higher binding strength for OPM-GMs was observed for both the $\mathrm{N}$ terminus $\left(K_{D}=424.5 \mu \mathrm{M}\right.$ and $\left.L=17.5\right)$ and the region spanning residues 65-97 $\left(K_{D}=3945 \mu \mathrm{M}\right.$ and $\left.L=3.8\right)$. CEST measurements indicated that the conformations of $\alpha \mathrm{S}$ at the surface of OPM-GMs feature a primary binding site in the $\mathrm{N}$-terminal region (residues 1 to 35 ), which binds with weaker affinity as observed in the case of IPM and SL-SUVs ${ }^{43}$ (Fig. 3b). In addition, a secondary binding region (residues 36-98) was observed with low affinity, followed by a C-terminal segment (residues 99-140) that lacks any interaction propensity for OPM-GMs. Taken together these data indicate that, in contexts associated with an accumulation of GMs in the membrane, including physiological lipid rafts as well as neurodegenerative conditions ${ }^{52}$, aS changes considerably its interaction properties with OPM surfaces.

Significant alterations in the binding properties were also observed in the case of IPM-GMs, particularly in the region 65-97, resulting in nearly a sixfold increase in the interaction propensity compared to IPM $\left(K_{D}=16.0 \mu \mathrm{M}\right.$ and $\left.L=34.5\right)$. By contrast, the N-terminal region of aS maintained similar strong binding for IPM-GMs $\left(K_{D}=5.8 \mu \mathrm{M}\right.$ and $\left.L=12.2\right)$ as for IPM (Fig. 3c). CEST analysis confirmed that most of the alterations in the residue-specific interactions occur in the region 65-97 of aS (Fig. 3d). The other regions of $\alpha \mathrm{S}$ were found to establish similar types of interactions with IPM and IPM-GMs.

We then probed whether the changes in binding propensity and conformational properties of $\alpha \mathrm{S}$ at the surface of IPM-GMs affect the mechanism of stabilisation of SL-SUVs docked onto the IPM surface (Fig. 3e, f). The measurements indicated a stronger 

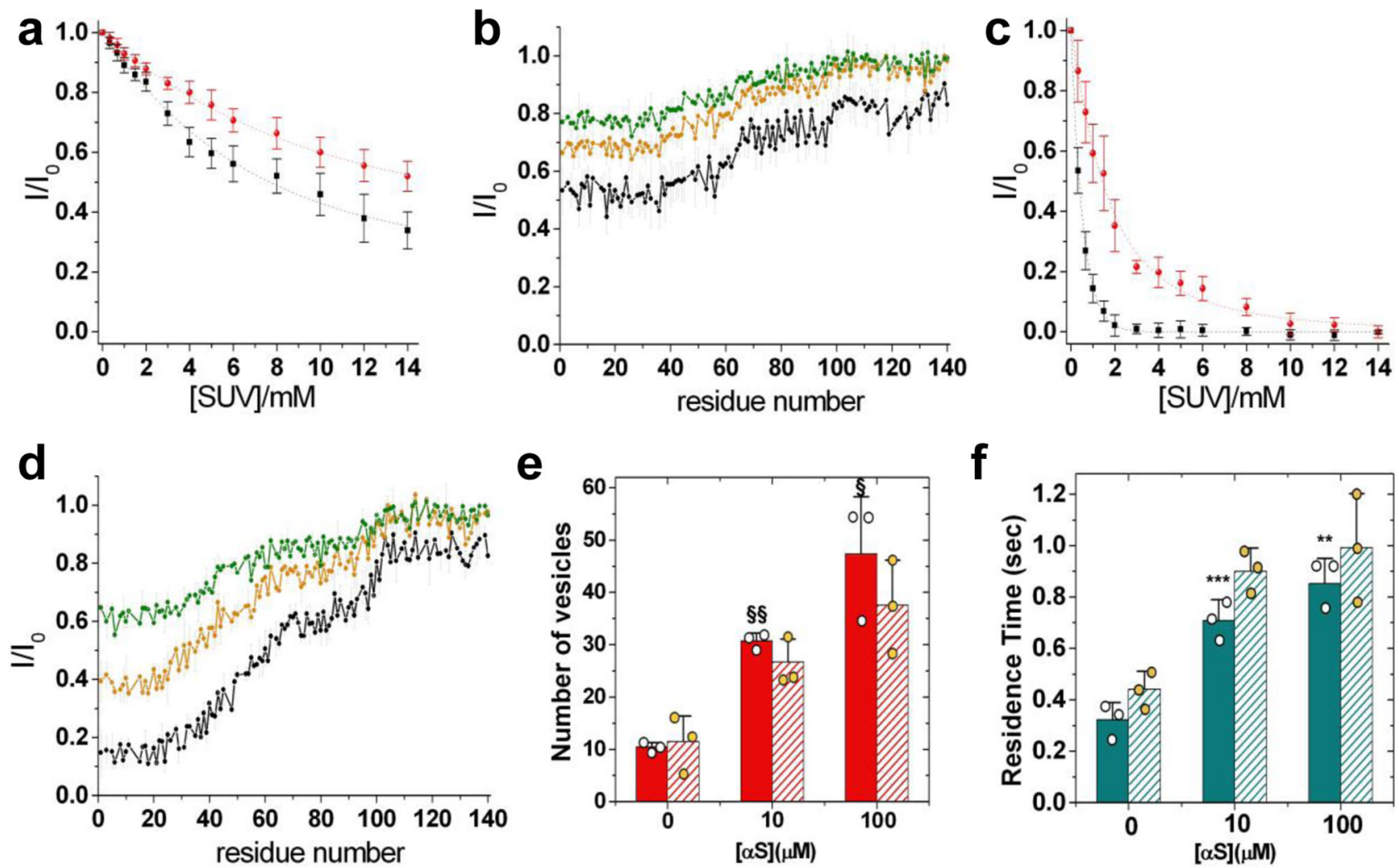

Fig. 3 Effect of GMs on the interaction of $\alpha \mathbf{S}$ with IPM and OPM. a Binding curves of $\alpha$ S to OPM-GMs SUVs monitored via the signal attenuation of the peaks in the ${ }^{1} \mathrm{H}-{ }^{15} \mathrm{~N}-\mathrm{HSQC}$ spectra of $\alpha \mathrm{S}(50 \mu \mathrm{M})$ as a function of the concentration of SUVs. The signal attenuations have been averaged over the individual residues of the $\mathrm{N}$-terminal region (black) and the region spanning residues 65-97 (red), and error bars report the standard deviation of these values. The resulting $K_{D}$ values are $424.5 \mu \mathrm{M}(L=17.5)$ and $3945 \mu \mathrm{M}(L=3.8)$ for the $\mathrm{N}$-terminal region and the region spanning residues $65-97$, respectively. b NMR CEST profiles along the sequence measured using a saturation bandwidth of $350 \mathrm{~Hz}$ (Fig. S5 for measurements performed with a bandwidth of $170 \mathrm{~Hz}$ ) for the interaction between $\alpha \mathrm{S}$ and OPM-GMs. Black, orange and green lines refer to the averaged CEST profiles measured using offsets at $\pm 1.5, \pm 3.0$, and $\pm 5.0 \mathrm{kHz}$, respectively. Error bars report the standard deviation estimated on the triplicate measurements. C Interaction between $\alpha \mathrm{S}$ and IPM-GMs (see (a) for details). The resulting binding affinities are $5.8 \mu \mathrm{M}(L=12.2)$ and $16.0 \mu \mathrm{M}(L=34.5)$ for the $\mathrm{N}$ terminus and the region 65-97, respectively. d NMR CEST analysis of the interaction between $\alpha \mathrm{S}$ and IPM-GMs measured using a saturation bandwidth of $350 \mathrm{~Hz}$ (Fig. S5 for measurements performed with a bandwidth of $170 \mathrm{~Hz}$ ). Details as in $\mathbf{b}$. Error bars report the standard deviation estimated on the triplicate measurements. e Statistical analysis of the number of docked vesicles on IPM-GM surfaces (filled bars) imaged in the focal plane at different concentrations of $\alpha \mathrm{S}$ and constant concentration of SUVs $(2 \mu \mathrm{M})$. For comparison, data measured using IPM are reported with striped bars. The symbols $\S$ and $\S \S$, indicate $p$ values of 0.028 and 0.0005 , respectively, calculated with the unpaired $t$ test using Welch's correction. Error bars report the standard deviation estimated on the triplicate measurements. $\mathbf{f}$ Residence times of docked vesicles on the IPM-GM surfaces (filled bars) at different concentrations of $\alpha \mathrm{S}$ and constant concentration of SUVs $(2 \mu \mathrm{M})$. For comparison, data measured using IPM are reported with striped bars. The symbols ${ }^{\star \star}$ and ${ }^{\star \star \star}$, indicate $p$ values of 0.02 and 0.03 , respectively, calculated with the unpaired $t$ test using Welch's correction. Error bars report the standard deviation estimated on the triplicate measurements.

stabilisation by aS of SL-SUVs docked onto IPM-GM surfaces, which is likely associated with an increase in the amount of $\alpha \mathrm{S}$ bound to IPM-GMs than in the case of IPM. The residence times of the vesicles, however, were found to be generally shorter compared to the case of IPM, likely because of the increased electrostatic repulsion between the two negatively charged membranes. Taken together, these data indicate that alterations in the composition of the IPM can affect the modes of stabilisation of docked SVs by aS.

\section{Discussion}

The biological activity of $a \mathrm{~S}$ is closely linked with the conformational properties of its membrane-bound state ${ }^{27}$. Interactions with synaptic membranes are indeed involved in most of the putative physiological functions of $a S^{6,25}$ and are crucial for determining the toxicity of its aberrant aggregates ${ }^{1,63,64}$. Although the most common membrane interaction by aS occurs in the context of SVs binding ${ }^{9-14}$, the binding plasticity of this disordered protein enables interactions with a variety of other cell membranes, including mitochondrial membranes and the $\mathrm{PM}^{26}$. In particular, it has been shown that monomeric forms of aS localises intracellularly near the $\mathrm{PM}^{65,66}$, and a number of studies have focused on the binding of the outer PM leaflet in the context of cellular uptake of $\alpha \mathrm{S}$ monomers ${ }^{59}$ and its pathological aggregates $^{1,67}$.

We showed here that the interactions of aS with the inner and outer leaflets of the PM are markedly different. The binding affinity for OPM was found to be negligible (Fig. 4a), however, upon increase of the GM content in the lipid composition, this interaction becomes considerably more favourable (Fig. 4b). This observation may be of relevance in the context of lipid rafts ${ }^{54}$ and some neurodegenerative conditions, both associated with increased GM content in the $\mathrm{PM}^{52}$. A different scenario resulted from the analysis of the interaction with IPM, showing moderately high binding affinity and unique conformational properties 


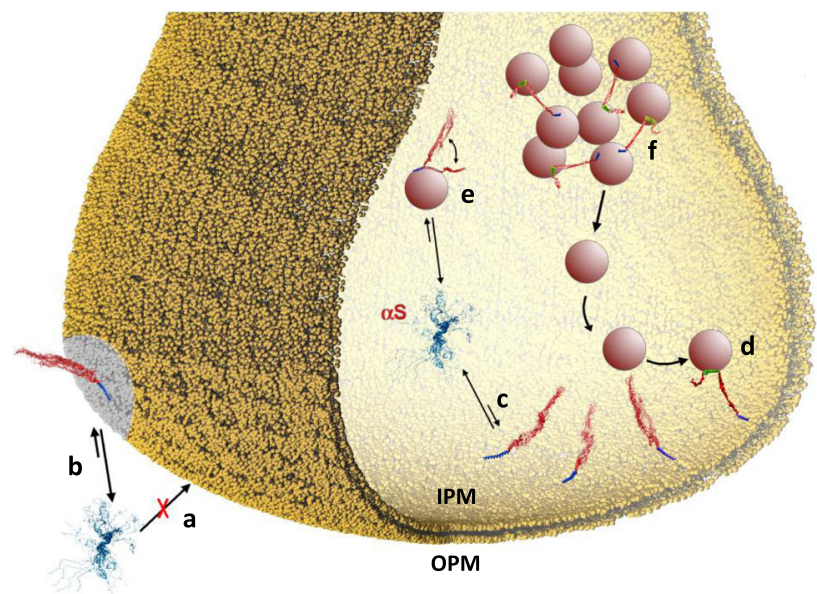

Fig. 4 Membrane trafficking of $\alpha \mathrm{S}$ at the synaptic termini. Schematic illustration of the different interactions between $\alpha S$ and biological membranes at the synaptic termini. a $\alpha S$ binds to OPM with negligible affinity. $\mathbf{b}$ When the content of GMs in the membrane composition increases, such as in the case of some neurodegenerative disorders 52 and in lipid rafts 54,65 , the affinity of $\alpha \mathrm{S}$ for OPM is considerably enhanced. $\mathbf{c}$ The binding affinity of $\alpha S$ for IPM is significantly higher than that observed in the case of OPM. Upon interaction with IPM, $\alpha$ S adopts a conformation where only the $\mathrm{N}$-terminal anchor (blue) is tightly bound to the membrane, with the region of residues 65-140 (red) having negligible association with the membrane surface. $\mathbf{d}$ This peculiar conformation has significant propensity to promote a double-anchor mechanism ( $\mathrm{N}$-terminal anchor in blue; second anchor spanning residues 65-97 in green) that stabilises the SV docking onto the IPM surface in an $\alpha S$ concentration-dependent manner. The IPM binding by $\alpha S$ competes with the binding to SVs (e), which is involved in the mainteinance of pools of vesicles (f) from which SVs diffuse toward the active zone.

in the membrane-bound state of $\mathrm{SS}$ (Fig. 4c). In particular, NMR measurements revealed that aS binds IPM primarily via its $\mathrm{N}$ terminal anchor, with negligible membrane interaction by most of the remainder of the protein, including the region 65-97. The weak membrane binding of this region may influence the behaviour of $\alpha \mathrm{S}$ at the surface of IPM, with particular relevance for the promotion of interactions between IPM and other synaptic membranes by means of the double-anchor mechanism ${ }^{17,43}$. The possibility that $\alpha \mathrm{S}$ could favour the docking of SVs to IPM has been discussed in literature ${ }^{15,68,69}$, and the present study, in addition to characterising the structural basis of this process, provides direct evidence that aS stabilises both thermodynamically (number of vesicles, Fig. $2 \mathrm{~d}$ ) and kinetically (residence time, Fig. 2e) the docking of SL-SUVs with IPM in a concentration-dependent manner.

There is general debate about the promotion or inhibition of SV exocytosis by $\alpha S^{15}$. It has been observed that $\alpha \mathrm{S}$ assists the formation of the SNARE complex via the direct interaction with synaptobrevin- $2^{9,10}$. Other studies, however, have suggested an inhibitory effect to the SNARE activity by this $\operatorname{protein}^{70-72}$. At the origin of these apparently contrasting results may be the promiscuity by which aS interacts with different membranes and proteins at the synaptic termini, often resulting in competing processes. For example the stabilisation of SV docking at IPM surfaces, which we here show being favoured by aS, competes with the SV binding and promotion of SV-SV clustering by this protein (Fig. 4e, f) ${ }^{14}$. The competition between these interactions can be regulated by a number of factors such as local bursts of calcium ions, which favour an extended double-anchor mechanism and mediate the localisation of $\alpha \mathrm{S}$ at presynaptic terminals ${ }^{16}$, post-translational modifications, including the phosphorylation of residues Ser $87^{73}$, Ser $129^{74}$ and Tyr $39^{68}$, and modifications of the IPM composition (Fig. 3e, f).

In conclusion, this study and the available literature provide evidence for a subtle equilibrium between various interactions by aS with lipid membranes in the presynaptic space that control its biological properties. A key role in this regulation is played by the equilibrium between membrane-bound and unbound states of the region 65-97 of $\alpha$, which overlaps with the amyloidogenic NAC region. The detachment from the membrane surface of this region influences the binding affinity of $\alpha \mathrm{S}$ for acidic lipid bilayers ${ }^{33}$ as well as the efficiency by which it promotes membrane-membrane interactions via the double-anchor mechanism ${ }^{17}$. In addition to having a regulatory role in the biological properties of aS, conformations exposing the region of residues 65-97 from the membrane surface can also trigger its self-assembly and aggregation. This region of aS, therefore, defines a tight balance between its functional and pathological states, and our results illustrate how the impairment of this balance in PD and related synucleinopathies could be expected to be inextricably linked to age-related changes in lipid homoeostasis.

\section{Methods}

$\boldsymbol{\alpha} \mathbf{S}$ purification. $\alpha \mathrm{S}$ was expressed and purified following an established protocol ${ }^{33}$ in which the protein was expressed in Escherichia coli using plasmid pT7-733. In order to obtain N-terminal acetylation of aS we used coexpression with a plasmid carrying the components of the NatB complex (Addgene) ${ }^{40}$. After transforming in BL21 (DE3)-gold cells (Agilent Technologies, Santa Clara, USA), uniformly ${ }^{15} \mathrm{~N}$ and/or ${ }^{13} \mathrm{C}$ labelled aS was obtained by growing the bacteria in isotope-enriched M9 minimal media containing $1 \mathrm{gl}^{-1}$ of ${ }^{15} \mathrm{~N}$ ammonium chloride, $2 \mathrm{gl}^{-1}$ of

${ }^{13} \mathrm{C}$-glucose (Sigma-Aldrich, St Louis, USA) and $1 \mathrm{~g}$ of protonated IsoGro ${ }^{15} \mathrm{~N}-{ }^{13} \mathrm{C}$ (Sigma, St. Louis, MO). The growth was obtained at $37^{\circ} \mathrm{C}$ under constant shaking at $250 \mathrm{rpm}$ and supplemented with $100 \mu \mathrm{g} \mathrm{ml}^{-1}$ ampicillin to an OD600 of 0.6. The expression was induced with $1 \mathrm{mM}$ isopropyl $\beta$-D-1-thiogalactopyranoside at $37^{\circ} \mathrm{C}$ for $4 \mathrm{~h}$, and the cells were harvested by centrifugation at $6200 \mathrm{~g}$ (Beckman Coulter, Brea, USA). The cell pellets were resuspended in lysis buffer $(10 \mathrm{mM}$ Tris- $\mathrm{HCl} \mathrm{pH}$ 8, $1 \mathrm{mM}$ EDTA and EDTA-free complete protease inhibitor cocktail tablets obtained from Roche, Basel, Switzerland) and lysed by sonication. The cell lysate was centrifuged at $22,000 \mathrm{~g}$ for $30 \mathrm{~min}$ to remove cell debris. In order to precipitate the heat-sensitive proteins, the supernatant was then heated for $20 \mathrm{~min}$ at $70^{\circ} \mathrm{C}$ and centrifuged at $22,000 \mathrm{~g}$. Subsequently streptomycin sulfate was added to the supernatant to a final concentration of $10 \mathrm{mg} \mathrm{ml}^{-1}$ to stimulate DNA precipitation. The mixture was stirred for $15 \mathrm{~min}$ at $4^{\circ} \mathrm{C}$ followed by centrifugation at 22,000 $\mathrm{g}$ Then, ammonium sulfate was added to the supernatant to a concentration of $360 \mathrm{mg} \mathrm{ml}^{-1}$ in order to precipitate the protein. The solution was stirred for $30 \mathrm{~min}$ at $4{ }^{\circ} \mathrm{C}$ and centrifuged again at $22,000 \mathrm{~g}$. The resulting pellet was resuspended in $25 \mathrm{mM}$ Tris- $\mathrm{HCl}, \mathrm{pH} 7.7$ and dialysed against the same buffer in order to remove salts. The dialysed solutions were then loaded onto an anion exchange column (26/10 Q sepharose high performance, GE Healthcare, Little Chalfont, UK) and eluted with a $0-1 \mathrm{M} \mathrm{NaCl}$ step gradient, and then further purified by loading onto a size exclusion column (Hiload 26/60 Superdex 75 preparation grade, GE Healthcare, Little Chalfont, UK). All the fractions containing the monomeric protein were pooled together and concentrated by using Vivaspin filter devices (Sartorius Stedim Biotech, Gottingen, Germany). The purity of the aliquots after each step was analyzed by SDS-PAGE and the protein concentration was determined from the absorbance at $275 \mathrm{~nm}$ using an extinction coefficient of $5600 \mathrm{M}^{-1} \mathrm{~cm}^{-1}$. Mass spectrometry was used to confirm that the level of $\mathrm{N}$-terminal acetylation was complete.

Preparation of SUVs. SUVs were prepared for the compositions of the inner and outer PM (IPM, OPM, IPM-GMs and OPM-GMs Table 1) using lipids purchased from Avanti Polar Lipids Inc. (Alabaster, USA) or Sigma-Aldrich (St Louis, USA) and mixed in chloroform solutions ${ }^{33,35}$. These include PC (Avanti code: 850457C), PE (Avanti code: $850757 \mathrm{C})$, PS (Avanti code: $840034 \mathrm{C}$ ), PI (Avanti code: 840042C), PIPs (Avanti code: 840045X), sphingomyelin (Avanti code: 860062C), cholesterol (Sigma code: C8667-1G), cerebrosides (Avanti code: 131303P), GM1 (Avanti code: 860065P) and GM3 (Avanti code: 860058P) (Table 1 for the molar fractions of the various lipid compositions $)^{41,42}$. In addition SL-SUVs containing $31 \% \mathrm{w} / \mathrm{w}$ of cholesterol (Sigma code: C8667-1G) were prepared using a mixture of DOPE (Avanti code: 850725C), DOPS (Avanti code: 840035C) and DOPC (Avanti code: $850375 \mathrm{C}$ ) at a ratio $5: 3: 2$ as previously described ${ }^{43}$. In order to employ the SL-SUVs in TIRF imaging, the mixture was doped with $0.1 \%$ of 1 -palmitoyl-2(dipyrrometheneboron difluoride) undecanoyl-sn-glycero-3-phosphocholine (Topfluor ${ }^{\circledast}$ P). Chloroform from the lipid mixture was evaporated under a stream of nitrogen gas and then dried thoroughly under vacuum to yield a thin lipid film. 
The dried thin film was then re-hydrated by adding aqueous buffer $(20 \mathrm{mM}$ sodium phosphate, $\mathrm{pH} 6.0)$ at a concentration of $10 \mathrm{mg} \mathrm{m}^{-1}$ (1.5\%) and subjected to vortex mixing. In all experiments described in this paper SUVs were obtained by using several cycles of freeze-thawing and sonication until the mixture became clear $^{33,35}$. In addition, an extrusion step was performed with a membrane with pores made of $50 \mathrm{~nm}$ diameter. All SUVs were controlled with DLS in order to obtain vesicles with the same average size.

\section{Chemical exchange saturation transfer NMR experiments. CEST} measurements $\mathrm{s}^{33,44-47}$ probed the equilibrium between membrane-unbound and membrane-bound states of $\alpha \mathrm{S}$ via direct detection of saturation in the resonances of the unbound state. In studying aS-SUV interactions, CEST shows higher sensitivity than measurements based on the signal attenuation in HSQC spectra, and as a result it enables measurements at low lipid:protein ratios to minimise $\alpha \mathrm{S}$ or lipid aggregation ${ }^{33}$. Moreover, CEST signals are directly sensitive to the interaction between $\alpha \mathrm{S}$ and the membrane surface and minimise the interference from additional factors that can contribute to the transverse relaxation rates of the protein resonances ${ }^{44-47}$. CEST measurements were carried at $283 \mathrm{~K}$ to minimise the reduction of the signal-to-noise in ${ }^{1} \mathrm{H}-{ }^{15} \mathrm{~N}$ correlations that is generated by the water exchange of amide protons ${ }^{33}$. aS samples $(300 \mu \mathrm{M})$ were incubated with the four types of SUVs $(0.6 \mathrm{mg} / \mathrm{ml})$ considered in this study (Table 1) in $20 \mathrm{mM}$ sodium phosphate buffer at $\mathrm{pH}$ 6.0, and NMR measurements were carried out using a Bruker spectrometer operating at a ${ }^{1} \mathrm{H}$ frequency of $700 \mathrm{MHz}$ and equipped with triple resonance HCN cryo-probe. The CEST experiments were based on ${ }^{1} \mathrm{H}-{ }^{15} \mathrm{~N}$ HSQC spectra and were carried out by applying constant wave saturation in the ${ }^{15} \mathrm{~N}$ channel. Assignment of the solution NMR resonances was obtained from our previous studies ${ }^{75}$ and controlled with a series of 3D spectra by following a published protocol ${ }^{76}$. Since we aimed at probing the exchange between monomeric $\alpha \mathrm{S}$ (having sharp resonances) and $\alpha \mathrm{S}$ bound to SUVs (having significantly broader resonances), a series of large offsets was employed $(-28,-21$, $-14,-9,-5,-3,-1.5,0,1.5,3,5,9,14,21$ and $28 \mathrm{kHz}$ ), resulting in CEST profiles of symmetrical shape $e^{33,44,45}$. An additional spectrum, saturated at $-100 \mathrm{kHz}$, was recorded as a reference. The CEST experiments were measured using a data matrix consisting of $2048\left(t_{2},{ }^{1} \mathrm{H}\right) \times 220\left(t_{1},{ }^{15} \mathrm{~N}\right)$ complex points. NMR spectra in this investigation were acquired using Topspin 3.6.0 (Bruker, AXS $\mathrm{GmBH}, \mathrm{DE})$ and processed with NMRpipe $10.9^{77}$ and Sparky 3.178.

Transverse relaxation NMR experiments. Standard pulse sequences were used for $T_{2}$ experiments ${ }^{79}$, including the watergate sequence ${ }^{80}$ to improve water suppression. $T_{2}$ values were obtained by fitting the experimental data with single exponential decays; the fitting of experimental data and the error analyses were performed with the programme Sparky $3.1^{78}$. Relaxation was measured at $10^{\circ} \mathrm{C}$ on a sample composed of aS $(300 \mu \mathrm{M})$ incubated with various types of SUVs (Table 1) at a concentration of $0.6 \mathrm{mg} / \mathrm{ml}$ and using a Bruker spectrometer operating at a ${ }^{1} \mathrm{H}$ frequency of $700 \mathrm{MHz}$ and equipped with triple resonance HCN cryo-probe. Assignment of the resonances as in the CEST measurements.

Total internal reflection fluorescence microscopy. TIRF microscopy can selectively excite fluorophore molecules within $150 \mathrm{~nm}$ from the surface of a support glass (cover slip) ${ }^{81}$. This technique increases the signal-to-noise and reduces the background fluorescence due to the minimisation of the excitation of fluorophores far from the cover slip ${ }^{82}$. This technique has been successfully applied to monitor properties of vesicles using sequential TIRF imaging ${ }^{83,84}$.We employed TIRF microscopy to image SL-SUVs docked onto the IPM (or IPM-GMs) surface. The experiments were made by creating an IPM (or IPM-GMs) lipid bilayer on an eight-well glass slide. The preparation of the glass slides included an incubation step of IPM or IPM-GMs SUVs overnight at $4^{\circ} \mathrm{C}$ to allow the vesicles to collapse onto the glass surfaces and form the bilayer. After the overnight incubation, the glass wells were gently washed with phosphate buffer to remove the lipids in excess. The IPM (or IPM-GMs) coated glass surfaces were then incubated with SL-SUVs labelled with $0.1 \%$ of Topfluor ${ }^{\circledR}$ PC lipids. Different concentrations of SL-SUVs were tested to find the right concentration to image single vesicles using TIRF. A total SL-SUVs concentration of $2 \mu \mathrm{M}$ was found to be optimal for single-molecule photobleaching experiments.

A custom TIRF imaging setup based on a Nikon Eclipse TE2000-U microscope (Nikon, Surrey, UK) was used. An argon ion laser (35LAP321-230, Melles Griot, USA) at $488 \mathrm{~nm}$ with $1.25 \mathrm{~mW}$ power was used for excitation. The images were acquired using an sCMOS camera (ORCA-Flash 4.0 V3 Digital CMOS camera, Hamamatsu Photonics, Japan). TIRF videos were obtained using $40 \mathrm{~ms}$ exposure time recordings with $4 \times 4$ binning and recorded continuously for 7500 frames (300 s). The last 3500 frames of the videos (140 s) were analysed to ensure that a steady state was reached after the initial step of photobleaching (160 s). All imaging experiments were performed in triplicates.

Particle analysis in TIRF imaging. TIRF images and videos were processed using the software Image $1.52 \mathrm{p}$ (NIH, USA ${ }^{85}$ and the TrackMate plugin ${ }^{86}$. To analyse the images, we first subtracted the background from each frame using the rolling ball algorithm and choosing a ball radius between 1 and 2 pixels. The background subtraction also enabled to remove particles associated with SUVs from the bulk solution that are not in focus, which appear as blurry particles with lower intensity of the sharp particles associated with docked vesicles in the TIRF focal plane. Particles in focus (docked vesicles) were tracked using a spot detection algorithm. To this end, we used TrackMate with the LoG (Laplacian of Gaussian) detector and set a particle diameter of 5 pixels, with a quality threshold value ranging between 8 and 10. This procedure provided an indexing of the particles including their $(x, y)$ coordinates in each frame of the TIRF videos. $P$ value (two-tailed) statistical analysis of TIRF data was performed using GraphPad Prism 9 (GraphPad software, USA) and calculated with the unpaired $t$ test using Welch's correction.

Residence time of SL-SUV docked onto IPM using TIRF experiments. The residence time of SL-SUVs docked onto IPM can be quantified by calculating the ACF from sequences of vesicles images ${ }^{87,88}$, which is defined as in Eq. 2 :

$$
P(\tau)=\sum_{t=0}^{T-\tau} \frac{1}{N_{t}} \sum_{i=1}^{N_{t}} \delta\left[w_{i}(t), w_{i}(t+\tau)\right]
$$

where $\tau$ is the characteristic time of the function and $t$ is the discrete time of the image sequence. $N_{\mathrm{t}}$ is the number of particles detected at time $t$ and $\delta\left[w_{i}(t), w_{i}(t+\tau)\right]$ assumes a value of 1 if the particle $i$ detected at time $t$ and at time $t+\tau$, and 0 otherwise. The ACF was fitted using a double exponential function (Eq. 3)

$$
P(\tau)=a_{1} e^{-\tau / t_{1}}+a_{2} e^{-\tau / t_{2}}+c_{0}
$$

Where the first coefficient $t_{1}$ is the fast decay time constant of the ACF, which can be attributed to the stochastic loss of correlation in the fluorescence intensity of the particles, and the second coefficient $t_{2}$ is the slow time constant directly associated to the residence time of the vesicles on the surface of IPM.

Dynamic light scattering (DLS). DLS measurements of vesicle size distributions were performed using a Zetasizer Nano ZSP instrument (Malvern Instruments, Malvern, UK) with backscatter detection at a scattering angle of $173^{\circ}$. The viscosity $(0.8882 \mathrm{cP})$ and the refractive index $(1.330)$ of water were used as parameters for the buffer solution, and the material properties of the analyte were set to those of the lipids (absorption coefficient of 0.001 and refractive index of 1.440). SUVs were used at a concentration of $0.05 \%$ in these measurements and the experiments were performed at $25^{\circ} \mathrm{C}$. The acquisition time for the collection of each dataset was $10 \mathrm{~s}$ and accumulation of the correlation curves was obtained using ten repetitions. Each measurement was repeated 10 times to estimate standard deviations and average values of the centres of the size distributions.

Circular dichroism analysis of $\alpha \mathrm{S}$ in the presence of different concentrations of SUVs. $\mathrm{CD}$ measurements were made at $10{ }^{\circ} \mathrm{C} . \mathrm{CD}$ samples were prepared in $20 \mathrm{mM}$ sodium phosphate buffer at $\mathrm{pH} 6.0$, by using a constant concentration of $\alpha \mathrm{S}$ $(10 \mu \mathrm{M})$ and variable concentrations of SUVs. Far-UV CD spectra were recorded on a JASCO J-810 equipped with a Peltier thermally controlled cuvette holder. Quartz cuvettes with path lengths of $1 \mathrm{~mm}$ were used, and CD spectra were obtained by averaging ten individual spectra recorded between 250 and $200 \mathrm{~nm}$ with a bandwidth of $1 \mathrm{~nm}$, a data pitch of $0.2 \mathrm{~nm}$, a scanning speed of $50 \mathrm{~nm} / \mathrm{min}$ and a response time of $4 \mathrm{~s}$. Each value of the $\mathrm{CD}$ signal intensity reported at $222 \mathrm{~nm}$ corresponds to the average of ten measurements. For each protein sample, the $\mathrm{CD}$ signal of the buffer used to solubilise the protein was recorded and subtracted from the $\mathrm{CD}$ signal of the protein. $\mathrm{CD}$ spectra in this investigation were acquired using Spectra Manager TM 2.8 (Jasco Research Ltd. CA).

Reporting summary. Further information on research design is available in the Nature Research Reporting Summary linked to this article.

\section{Data availability}

Data supporting the findings of this study are available within the article and its Supplementary Information Files and from the corresponding author on request. Python processing scripts can be found at https://github.com/vrettasm/analysis_code_Man_ et_al. Source data are provided with this paper.

Received: 30 April 2020; Accepted: 7 January 2021; Published online: 10 February 2021

\section{References}

1. Fusco, G. et al. Structural basis of membrane disruption and cellular toxicity by alpha-synuclein oligomers. Science 358, 1440-1443 (2017).

2. Luk, K. C. et al. Pathological alpha-synuclein transmission initiates Parkinsonlike neurodegeneration in nontransgenic mice. Science 338, 949-953 (2012). 
3. Chiti, F. \& Dobson, C. M. Protein misfolding, amyloid formation, and human disease: a summary of progress over the last decade. Annu Rev. Biochem 86, 27-68 (2017).

4. Lashuel, H. A., Overk, C. R., Oueslati, A. \& Masliah, E. The many faces of alpha-synuclein: from structure and toxicity to therapeutic target. Nat. Rev. Neurosci. 14, 38-48 (2013).

5. Uversky, V. N. \& Eliezer, D. Biophysics of Parkinson's disease: structure and aggregation of alpha-synuclein. Curr. Protein Pept. Sci. 10, 483-499 (2009).

6. Burre, J. The synaptic function of alpha-synuclein. J. Parkinsons Dis. 5, 699-713 (2015).

7. Murphy, D. D., Rueter, S. M., Trojanowski, J. Q. \& Lee, V. M. Synucleins are developmentally expressed, and alpha-synuclein regulates the size of the presynaptic vesicular pool in primary hippocampal neurons. J. Neurosci. 20, 3214-3220 (2000).

8. George, J. M., Jin, H., Woods, W. S. \& Clayton, D. F. Characterization of a novel protein regulated during the critical period for song learning in the zebra finch. Neuron 15, 361-372 (1995).

9. Burre, J., Sharma, M. \& Sudhof, T. C. Alpha-synuclein assembles into higherorder multimers upon membrane binding to promote SNARE complex formation. Proc. Natl Acad. Sci. USA 111, E4274-E4283 (2014).

10. Burre, J. et al. Alpha-synuclein promotes SNARE-complex assembly in vivo and in vitro. Science 329, 1663-1667 (2010).

11. Auluck, P. K., Caraveo, G. \& Lindquist, S. Alpha-synuclein: membrane interactions and toxicity in Parkinson's disease. Annu Rev. Cell Dev. Biol. 26, 211-233 (2010).

12. Soper, J. H. et al. Alpha-synuclein-induced aggregation of cytoplasmic vesicles in Saccharomyces cerevisiae. Mol. Biol. Cell 19, 1093-1103 (2008).

13. Gitler, A. D. et al. The Parkinson's disease protein alpha-synuclein disrupts cellular Rab homeostasis. Proc. Natl Acad. Sci. USA 105, 145-150 (2008).

14. Diao, J. et al. Native alpha-synuclein induces clustering of synaptic-vesicle mimics via binding to phospholipids and synaptobrevin-2/VAMP2. Elife 2, e00592 (2013).

15. Lautenschlager, J., Kaminski, C. F. \& Kaminski Schierle, G. S. Alpha-synuclein -regulator of exocytosis, endocytosis, or both? Trends Cell Biol. 27, 468-479 (2017).

16. Lautenschlager, J. et al. C-terminal calcium binding of alpha-synuclein modulates synaptic vesicle interaction. Nat. Commun. 9, 712 (2018).

17. Fusco, G. et al. Structural basis of synaptic vesicle assembly promoted by asynuclein. Nat. Commun. 7, 12563 (2016).

18. Vargas, K. J. et al. Synucleins regulate the kinetics of synaptic vesicle endocytosis. J. Neurosci. 34, 9364-9376 (2014)

19. Nemani, V. M. et al. Increased expression of alpha-synuclein reduces neurotransmitter release by inhibiting synaptic vesicle reclustering after endocytosis. Neuron 65, 66-79 (2010).

20. Cooper, A. A. et al. Alpha-synuclein blocks ER-Golgi traffic and Rab1 rescues neuron loss in Parkinson's models. Science 313, 324-328 (2006).

21. Plotegher, N., Gratton, E. \& Bubacco, L. Number and brightness analysis of alpha-synuclein oligomerization and the associated mitochondrial morphology alterations in live cells. Biochim. Biophys. Acta 1840, 2014-2024 (2014).

22. Ramezani, M. et al. Regulation of exocytosis and mitochondrial relocalization by alpha-synuclein in a mammalian cell model. NPJ Parkinsons Dis. 5, 12 (2019).

23. Menges, S. et al. Alpha-synuclein prevents the formation of spherical mitochondria and apoptosis under oxidative stress. Sci. Rep. 7, 42942 (2017).

24. Maltsev, A. S., Chen, J., Levine, R. L. \& Bax, A. Site-specific interaction between alpha-synuclein and membranes probed by NMR-observed methionine oxidation rates. J. Am. Chem. Soc. 135, 2943-2946 (2013).

25. Fusco, G. \& Sanz-Hernandez, M. Order and disorder in the physiological membrane binding of alpha-synuclein. Curr. Opin. Struct. Biol. 48, 49-57 (2018).

26. Snead, D. \& Eliezer, D. Alpha-synuclein function and dysfunction on cellular membranes. Exp. Neurobiol. 23, 292-313 (2014).

27. Newberry, R. W., Leong, J. T., Chow, E. D., Kampmann, M. \& DeGrado, W. F. Deep mutational scanning reveals the structural basis for alpha-synuclein activity. Nat. Chem. Biol. 16, 653-659 (2020).

28. Zhu, M. \& Fink, A. L. Lipid binding inhibits alpha-synuclein fibril formation. J. Biol. Chem. 278, 16873-16877 (2003).

29. Perrin, R. J., Woods, W. S., Clayton, D. F. \& George, J. M. Exposure to long chain polyunsaturated fatty acids triggers rapid multimerization of synucleins. J. Biol. Chem. 276, 41958-41962 (2001).

30. Lorenzen, N., Lemminger, L., Pedersen, J. N., Nielsen, S. B. \& Otzen, D. E. The $\mathrm{N}$-terminus of alpha-synuclein is essential for both monomeric and oligomeric interactions with membranes. FEBS Lett. 588, 497-502 (2014).

31. Winner, B. et al. In vivo demonstration that alpha-synuclein oligomers are toxic. Proc. Natl Acad. Sci. USA 108, 4194-4199 (2011).

32. Theillet, F. X. et al. Structural disorder of monomeric alpha-synuclein persists in mammalian cells. Nature 530, 45-50 (2016).
33. Fusco, G. et al. G. Direct observation of the three regions in alpha-synuclein that determine its membrane-bound behaviour. Nat. Commun. 5, 3827 (2014).

34. Maltsev, A. S., Ying, J. \& Bax, A. Impact of N-terminal acetylation of alphasynuclein on its random coil and lipid binding properties. Biochemistry 51, 5004-5013 (2012).

35. Bodner, C. R., Dobson, C. M. \& Bax, A. Multiple tight phospholipid-binding modes of alpha-synuclein revealed by solution NMR spectroscopy. J. Mol. Biol. 390, 775-790 (2009).

36. Ulmer, T. S. \& Bax, A. Comparison of structure and dynamics of micellebound human alpha-synuclein and Parkinson disease variants. J. Biol. Chem. 280, 43179-43187 (2005).

37. Eliezer, D., Kutluay, E., Bussell, R. Jr. \& Browne, G. Conformational properties of alpha-synuclein in its free and lipid-associated states. J. Mol. Biol. 307, 1061-1073 (2001)

38. Ulmer, T. S., Bax, A., Cole, N. B. \& Nussbaum, R. L. Structure and dynamics of micelle-bound human alpha-synuclein. J. Biol. Chem. 280, 9595-9603 (2005).

39. Cheng, C. Y., Varkey, J., Ambroso, M. R., Langen, R. \& Han, S. Hydration dynamics as an intrinsic ruler for refining protein structure at lipid membrane interfaces. Proc. Natl Acad. Sci. USA 110, 16838-16843 (2013).

40. Runfola, M. et al. The N-terminal acetylation of alpha-synuclein changes the affinity for lipid membranes but not the structural properties of the bound state. Sci. Rep. 10, 204 (2020)

41. Ingolfsson, H. I. et al. Computational lipidomics of the neuronal plasma membrane. Biophys. J. 113, 2271-2280 (2017).

42. van Meer, G., Voelker, D. R. \& Feigenson, G. W. Membrane lipids: where they are and how they behave. Nat. Rev. Mol. Cell Biol. 9, 112-124 (2008).

43. Man, W. K. et al. Role of cholesterol in modulating the binding of alphasynuclein to synaptic-like vesicles. Front. Neurosci. 14, 18 (2020).

44. Huang, H., Milojevic, J. \& Melacini, G. Analysis and optimization of saturation transfer difference NMR experiments designed to map early selfassociation events in amyloidogenic peptides. J. Phys. Chem. B 112, 5795-5802 (2008).

45. Fawzi, N. L., Ying, J., Ghirlando, R., Torchia, D. A. \& Clore, G. M. Atomicresolution dynamics on the surface of amyloid-beta protofibrils probed by solution NMR. Nature 480, 268-272 (2011).

46. Vallurupalli, P., Bouvignies, G. \& Kay, L. E. Studying “invisible” excited protein states in slow exchange with a major state conformation. J. Am. Chem. Soc. 134, 8148-8161 (2012).

47. Milojevic, J., Esposito, V., Das, R. \& Melacini, G. Understanding the molecular basis for the inhibition of the Alzheimer's Abeta-peptide oligomerization by human serum albumin using saturation transfer difference and off-resonance relaxation NMR spectroscopy. J. Am. Chem. Soc. 129, 4282-4290 (2007).

48. Fusco, G. et al. Structural ensembles of membrane-bound alpha-synuclein reveal the molecular determinants of synaptic vesicle affinity. Sci. Rep. 6 , 27125 (2016).

49. Ueda, K. et al. Molecular cloning of cDNA encoding an unrecognized component of amyloid in Alzheimer disease. Proc. Natl Acad. Sci. USA 90 11282-11286 (1993)

50. Galvagnion, C. et al. Lipid vesicles trigger alpha-synuclein aggregation by stimulating primary nucleation. Nat. Chem. Biol. 11, 229-234 (2015).

51. Pernber, Z., Blennow, K., Bogdanovic, N., Mansson, J. E. \& Blomqvist, M. Altered distribution of the gangliosides GM1 and GM2 in Alzheimer's disease. Dement Geriatr. Cogn. Disord. 33, 174-188 (2012).

52. Yamamoto, N., Matsubara, T., Sato, T. \& Yanagisawa, K. Age-dependent high density clustering of GM1 ganglioside at presynaptic neuritic terminals promotes amyloid beta-protein fibrillogenesis. Biochim Biophys. Acta 1778, 2717-2726 (2008)

53. Molander-Melin, M. et al. Structural membrane alterations in Alzheimer brains found to be associated with regional disease development; increased density of gangliosides GM1 and GM2 and loss of cholesterol in detergentresistant membrane domains. J. Neurochem. 92, 171-182 (2005).

54. Liu, L., Zhang, K., Tan, L., Chen, Y. H. \& Cao, Y. P. Alterations in cholestero and ganglioside GM1 content of lipid rafts in platelets from patients with Alzheimer disease. Alzheimer Dis. Assoc. Disord. 29, 63-69 (2015).

55. Evangelisti, E. et al. Chiti, F. \& Stefani, M. Membrane lipid composition and its physicochemical properties define cell vulnerability to aberrant protein oligomers. J. Cell Sci. 125, 2416-2427 (2012).

56. Martinez, Z., Zhu, M., Han, S. \& Fink, A. L. GM1 specifically interacts with alpha-synuclein and inhibits fibrillation. Biochemistry 46, 1868-1877 (2007).

57. Perissinotto, F. et al. GM1 ganglioside role in the interaction of alphasynuclein with lipid membranes: morphology and structure. Biophys. Chem. 255, 106272 (2019)

58. Kaur, U. \& Lee, J. C. Unroofing site-specific a-synuclein-lipid interactions at the plasma membrane. Proc. Natl Acad. Sci. 117, 18977-18983 (2020).

59. Park, J. Y. et al. On the mechanism of internalization of alpha-synuclein into microglia: roles of ganglioside GM1 and lipid raft. J. Neurochem. 110, 400-411 (2009). 
60. Gaspar, R., Pallbo, J., Weininger, U., Linse, S. \& Sparr, E. Ganglioside lipids accelerate alpha-synuclein amyloid formation. Biochim Biophys Acta. 1866, 1062-1072 (2018).

61. Grey, M. et al. Acceleration of alpha-synuclein aggregation by exosomes. $J$. Biol. Chem. 290, 2969-2982 (2015).

62. Chiricozzi, E., et al. GM1 ganglioside is a key factor in maintaining the mammalian neuronal functions avoiding neurodegeneration. Int. J. Mol. Sci. 21, 868 (2020).

63. Stok, R. \& Ashkenazi, A. Lipids as the key to understanding alpha-synuclein behaviour in Parkinson disease. Nat Rev Mol Cell Biol. 21, 357-335 (2020).

64. Cascella, R. et al. Probing the origin of the toxicity of oligomeric aggregates of alpha-synuclein with antibodies. ACS Chem. Biol. 14, 1352-1362 (2019).

65. Fortin, D. L. et al. Lipid rafts mediate the synaptic localization of alphasynuclein. J. Neurosci. 24, 6715-6723 (2004).

66. Outeiro, T. F. \& Lindquist, S. Yeast cells provide insight into alpha-synuclein biology and pathobiology. Science 302, 1772-1775 (2003).

67. Masaracchia, C. et al. Membrane binding, internalization, and sorting of alpha-synuclein in the cell. Acta Neuropathol. Commun. 6, 79 (2018).

68. Dikiy, I. et al. Semisynthetic and in vitro phosphorylation of alpha-synuclein at Y39 promotes functional partly helical membrane-bound states resembling those induced by PD mutations. ACS Chem. Biol. 11, 2428-2437 (2016).

69. Dikiy, I. \& Eliezer, D. Folding and misfolding of alpha-synuclein on membranes. Biochim Biophys. Acta 1013-8, 2012 (1818).

70. Thayanidhi, N. et al. Alpha-synuclein delays endoplasmic reticulum (ER)-toGolgi transport in mammalian cells by antagonizing ER/Golgi SNAREs. Mol. Biol. Cell 21, 1850-1863 (2010).

71. Lai, Y. et al. Nonaggregated alpha-synuclein influences SNARE-dependent vesicle docking via membrane binding. Biochemistry 53, 3889-3896 (2014).

72. DeWitt, D. C. \& Rhoades, E. alpha-Synuclein can inhibit SNARE-mediated vesicle fusion through direct interactions with lipid bilayers. Biochemistry 52, 2385-2387 (2013).

73. Oueslati, A., Paleologou, K. E., Schneider, B. L., Aebischer, P. \& Lashuel, H. A. Mimicking phosphorylation at serine 87 inhibits the aggregation of human alpha-synuclein and protects against its toxicity in a rat model of Parkinson's disease. J. Neurosci. 32, 1536-1544 (2012).

74. Stephens, A. D. et al. Extent of N-terminus exposure by altered long-range interactions of monomeric alpha-synuclein determines its aggregation propensity. Nat. Commun. 10, 1038 (2020).

75. Fusco, G., Sanz-Hernandez, M., Ruggeri, F. S., Vendruscolo, M., Dobson, C. M. \& De Simone, A. Molecular determinants of the interaction of EGCG with ordered and disordered proteins. Biopolymers 109, e23117 (2018).

76. Fusco, G. et al. C.M. (1) H, (1)(3)C and (1)(5) N resonance assignments of human muscle acylphosphatase. Biomol. NMR Assign. 6, 27-29 (2012).

77. Delaglio, F. et al. NMRPipe: a multidimensional spectral processing system based on UNIX pipes. J. Biomol. NMR 6, 277-293 (1995).

78. Lee, W., Tonelli, M. \& Markley, J. L. NMRFAM-SPARKY: enhanced software for biomolecular NMR spectroscopy. Bioinformatics 31, 1325-1327 (2015).

79. Farrow, N. A. et al. Backbone dynamics of a free and phosphopeptidecomplexed Src homology 2 domain studied by $15 \mathrm{~N}$ NMR relaxation. Biochemistry 33, 5984-6003 (1994).

80. Piotto, M., Saudek, V. \& Sklenar, V. Gradient-tailored excitation for singlequantum NMR spectroscopy of aqueous solutions. J. Biomol. NMR 2, 661-665 (1992).

81. Lin, J. \& Hoppe, A. D. Uniform total internal reflection fluorescence illumination enables live cell fluorescence resonance energy transfer microscopy. Microsc Microanal. 19, 350-359 (2013).

82. Mattheyses, A. L., Simon, S. M. \& Rappoport, J. Z. Imaging with total internal reflection fluorescence microscopy for the cell biologist. J. Cell Sci. 123 , 3621-3628 (2010).

83. Flagmeier, $\mathrm{P}$. et al. Ultrasensitive measurement of $\mathrm{Ca}(2+)$ influx into lipid vesicles induced by protein aggregates. Angew. Chem. Int Ed. Engl. 56, 7750-7754 (2017).
84. Akopova, I. et al. Imaging exocytosis of ATP-containing vesicles with TIRF microscopy in lung epithelial A549 cells. Purinergic Signal 8, 59-70 (2012).

85. Schneider, C. A., Rasband, W. S. \& Eliceiri, K. W. NIH image to imageJ: 25 years of image analysis. Nat. Methods 9, 671-675 (2012).

86. Tinevez, J. Y. et al. TrackMate: an open and extensible platform for singleparticle tracking. Methods 115, 80-90 (2017).

87. Liu, J., Sun, Y., Qi, J. \& Marcu, L. A novel method for fast and robust estimation of fluorescence decay dynamics using constrained least-squares deconvolution with Laguerre expansion. Phys. Med Biol. 57, 843-865 (2012).

88. Yang, H. et al. Protein conformational dynamics probed by single-molecule electron transfer. Science 302, 262-266 (2003).

\section{Acknowledgements}

This research is supported by the European Research Council (ERC) Consolidator Grant (CoG) 'BioDisOrder" (819644, A.D.S.), the UK Medical Research Council (MR/ N000676/1, A.D. and M.V. and MR/R000255/1, A.D.S.), the Centre for Misfolding Diseases of the University of Cambridge (M.V.), the St John's College Fellowship (G.F.) and Leverhulme Trust grant (RPG-2015-345, B.T. and L.Y).

\section{Author contributions}

A.D. and G.F. designed the research. W.K.M., B.T., S.P., G.F. and A.D. performed the experiments. W.K.M., B.T., M.D.V., L.Y., A.D. and G.F. analysed the data., M.V., G.F. and A.D., wrote the manuscript. All authors revised the manuscript critically for important intellectual content and approved the final version.

\section{Competing interests}

The authors declare no competing interests.

\section{Additional information}

Supplementary information The online version contains supplementary material available at https://doi.org/10.1038/s41467-021-21027-4.

Correspondence and requests for materials should be addressed to A.D.S. or G.F.

Peer review information Nature Communications thanks Luigi Bubacco and William DeGrado for their contribution to the peer review of this work. Peer reviewer reports are available.

Reprints and permission information is available at http://www.nature.com/reprints

Publisher's note Springer Nature remains neutral with regard to jurisdictional claims in published maps and institutional affiliations.

Open Access This article is licensed under a Creative Commons Attribution 4.0 International License, which permits use, sharing, adaptation, distribution and reproduction in any medium or format, as long as you give appropriate credit to the original author(s) and the source, provide a link to the Creative Commons license, and indicate if changes were made. The images or other third party material in this article are included in the article's Creative Commons license, unless indicated otherwise in a credit line to the material. If material is not included in the article's Creative Commons license and your intended use is not permitted by statutory regulation or exceeds the permitted use, you will need to obtain permission directly from the copyright holder. To view a copy of this license, visit http://creativecommons.org/ licenses/by/4.0/

(C) The Author(s) 2021 